\title{
The behavior of residual pulmonary artery gradient after arterial switch operation: A longitudinal data analysis
}

\author{
HANI ALKATTAN ${ }^{1}$, Obayda M Diraneyya ${ }^{1}$, Hatem Elmontaser ${ }^{1}$, Joohum Jaweed ${ }^{1}$, \\ Abdulsalam Alsayad ${ }^{1}$, Ahmed Arifi ${ }^{1}$, and Abdullah Alghamdi ${ }^{2}$ \\ ${ }^{1}$ National Guard Health Affairs \\ ${ }^{2}$ King Abdulaziz Cardiac Center
}

July 2, 2020

\begin{abstract}
Objective: The arterial switch operation is the standard treatment for the transposition of the great arteries. The timely variation in the residual pressure gradient across the pulmonary arteries is ill-defined. This work is aimed to study the progressive changes in the pressure gradient across the pulmonary valve and pulmonary arteries after arterial switch operation (ASO). Methods: All eligible patients for this study who underwent arterial switch operation between 2000 and 2019 were reviewed. Transthoracic echocardiography (TTE), was used to estimate the peak pressure gradient across the pulmonary artery and its branches. The primary outcome was the total peak pressure gradient (TPG) which is the sum of peak pressure gradients across the main pulmonary artery and pulmonary artery branches. Furthermore, a longitudinal data analyses with mixed effect modeling were used to determine the independent predictors for the changes in pressure gradient. Results: 309 patients were included in the study. Over 17-year follow up, the freedom from pulmonary stenosis reintervention was $95 \%$ (16 out of the 309 patients underwent reintervention $=5 \%$ ). the Longitudinal data analyses of serial 1844 echocardiographic studies for the included patients revealed that the TPG recorded in the first postoperative echocardiogram across pulmonary valve, right and left pulmonary artery branches was the most significant predictor for reintervention. Conclusion: The total peak gradient measured in the first postoperative echocardiogram is the most important predictor for reintervention. We propose that a total peak gradient in the first postoperative echocardiography of $55 \mathrm{mmHg}$ or more is a predictor for reintervention
\end{abstract}

The behavior of residual pulmonary artery gradient after arterial switch operation:

\section{A longitudinal data analysis}

\section{ABSTRACT}

Objective: The arterial switch operation is the standard treatment for the transposition of the great arteries. The timely variation in the residual pressure gradient across the pulmonary arteries is ill-defined. This work is aimed to study the progressive changes in the pressure gradient across the pulmonary valve and pulmonary arteries after arterial switch operation (ASO).

Methods: All eligible patients for this study who underwent arterial switch operation between 2000 and 2019 were reviewed. Transthoracic echocardiography (TTE), was used to estimate the peak pressure gradient across the pulmonary artery and its branches. The primary outcome was the total peak pressure gradient (TPG) which is the sum of peak pressure gradients across the main pulmonary artery and pulmonary artery branches. Furthermore, a longitudinal data analyses with mixed effect modeling were used to determine the independent predictors for the changes in pressure gradient.

Results: 309 patients were included in the study. Over 17-year follow up, the freedom from pulmonary stenosis reintervention was $95 \%$ ( 16 out of the 309 patients underwent reintervention $=5 \%$ ). the Longitudinal 
data analyses of serial 1844 echocardiographic studies for the included patients revealed that the TPG recorded in the first postoperative echocardiogram across pulmonary valve, right and left pulmonary artery branches was the most significant predictor for reintervention.

Conclusion: The total peak gradient measured in the first postoperative echocardiogram is the most important predictor for reintervention. We propose that a total peak gradient in the first postoperative echocardiography of $55 \mathrm{mmHg}$ or more is a predictor for reintervention.

\section{Main Document}

\section{INTRODUCTION:}

Transposition of the great arteries (TGA) is a common cyanotic congenital heart disease either in isolation or as a part of more complex congenital cardiac anomalies. Since its inception in 1975 by Jatene, the arterial switch operation (ASO) has become the standard method for surgical repair of the TGA ${ }^{1,2}$.

The goal of surgical repair is to have an anatomical correction. Part of the surgical treatment is performing the Lecompte procedure where the pulmonary arteries are relocated anteriorly ${ }^{4}$. Furthermore, the neopulmonary artery is reconstructed, most often using a pericardial patch. Although the current operative mortality is low and the mid and late outcomes are satisfactory, there are still some complications, one of which is the residual pressure gradient across the pulmonary valve and reconstructed pulmonary arteries ${ }^{3-6}$.

The pressure gradient across the neo-pulmonary artery (especially in the supra-valvular area) is the most crucial determinant for reintervention, though modifications of arterial switch operation have reduced the occurrence of pulmonary stenosis ${ }^{4,5,7}$.

The reported incidence of reoperation related to pulmonary stenosis after the arterial switch operation ranges from $5-30 \%^{5,8-10}$. Because the pulmonary artery pressure gradient can vary with the somatic growth, we sought to examine the behavior of the pulmonary artery pressure gradient over time and to determine the contributing factors and its relationship with the need for reintervention.

\section{MATERIAL AND METHODS:}

\section{Study Population:}

After approval of the research ethics board, all consecutive patients who underwent ASO between 2000 and 2019 were reviewed. Exclusion criteria were: double outlet right ventricle (DORV), congenitally corrected transposition of the great arteries (CCTGA), specific pathology forms of transpositions (e.g., isomerism, posterior transposition), late presenting patients (age more than 3-months) and patients who died early (in-hospital death). These exclusion criteria were applied to yield a homogenous group of patients.

\section{Institutional Surgical Technique:}

Our institutional repair strategy for the transposition of the great arteries includes performing LeCompte maneuver and reconstruction of the neo-pulmonary artery using quadrangular fresh autologous pericardial patch. The pulmonary reconstruction technique includes mobilization of both pulmonary artery branches into the hila, direct suturing of the patch to $2 / 3$ of the annulus of the neo-pulmonary trunk, and re-suspension of the neo-pulmonary valve inside the patch. An anastomosis without tension is then performed, using the reconstructed pulmonary tract to fit out the expected size of the distal-pulmonary artery (Figure 1).

\section{Echocardiographic follow up:}

All included patients underwent Intraoperative transesophageal echocardiogram (TEE) as well as a routine pre-discharge TTE (first post-operative echocardiogram). Patient follow-up and need for reintervention were determined from the outpatient clinic charts. The TTE was performed regularly in the outpatient setting to assess the pressure gradient across the main pulmonary artery and the pulmonary artery branches. In our center the follow up protocol is to do TTE after 6 weeks, 3 months, 6 months, 1 year of discharge, then once yearly unless there are pathologic changes in first year of follow up. Follow up was available for all patients 
at a mean duration of 9.3 years. the follow-up included for the purpose of this study, the valvular function and the pulmonary artery pressure gradient.

\section{Outcomes:}

The primary outcome was the total peak gradient across the main pulmonary artery (MPA), right pulmonary artery (RPA), and left pulmonary artery (LPA); thereby, total peak gradient $(\mathrm{TPG})=\mathrm{MPA}+\mathrm{RPA}+\mathrm{LPA}$ measured by echocardiography in the postoperative period (pre-discharge echocardiogram). The secondary outcome was the reintervention by means of percutaneous or surgical intervention.

\section{Statistics:}

Continuous data were presented as means with standard deviation and categorical data were presented as proportions. Kaplan-Meier statistics were used to display time to reintervention. A multivariable proportional hazard modeling was used to identify the influence of preoperative and operative variables on the need for reintervention. Longitudinal data analyses with mixed effect modeling were used to display and identify predictors of the progressive changes in the pressure gradient following repair. The statistical software system SAS, version 9.4, Cary, USA, was used for all statistical analyses.

\section{Results:}

\section{Study patients}

A total of 411 patients who underwent arterial switch operations between 2000 and 2019 were reviewed. The inclusion/exclusion criteria were applied, 67 patients had specific pathology requiring arterial switch operation were excluded because this pathology had innate pulmonary arteries disease, the rest are 344 patients, 24 of them who were older than 90 days were also excluded due to basic significant pulmonary hypertension in this population. There were 11 early mortalities. There were no late mortalities. Most of the mortalities were in the early era of the study. None of those (mortality group of patients) underwent reintervention for residual pulmonary artery stenosis. Therefore, the final study cohort included 309 patients (Figure 2).

Baseline characteristics:

$35 \%$ of patients are male, $65 \%$ of patients had simple transposition and $35 \%$ had complex transposition, balloon atrial septostomy (BAS) was done for $55 \%$ of patients, mean age of the

population was $20 \pm 17$ days, mean weight was $3.2 \pm 0.5 \mathrm{~kg}$, mean height was $51 \pm 5 \mathrm{~cm}$, mean body surface area (BSA) was $0.2 \pm 0.02$, mean cardio-pulmonary bypass (CPB) time was $160 \pm 39$ minutes, mean cross clamp (CC) time was $95 \pm 27$ minutes. Mean PG across the main pulmonary artery, left PA, Right PA are 9 \pm 7 , $14 \pm 11,17 \pm 13 \mathrm{mmHg}$ Respectively. The baseline characteristics are summarized in table 1 and 2 .

Comparison based on reintervention and preoperative variables

A univariable comparison was performed to identify the significant preoperative variables between those who underwent reintervention and no intervention group, (Table $3 \& 4$ ).

Age, weight, height, BSA, CPB time, CC time, gender, and surgeon there were not statistically different between both groups. However, the estimated PG across left PA, right PA, and TPG were statistically different.

Time to reintervention analysis

Time to reintervention using Kaplan-Meier statistics for the entire cohort of 309 patients showed 16 events (re-interventions) with freedom from re-intervention along the span of the study of 17 -year was $95 \%$. The mean follow-up time was 9.3 years (Figure 3). Survival analysis with stratification by the sex, surgeon, type of transposition and balloon atrial septostomy using log-rank statistics showed no significant differences. 
Serial Transthoracic Echocardiographic Measurements:

All patients underwent serial transthoracic echocardiographic evaluations. The minimum number of echocardiograms done for each patient (excluding the intraoperative TEE) was 1, and the maximum was $17($ mean $=6 \pm 4)$, Figure 4 .

Reintervention group $(\mathrm{N}=16)$ :

Of the 16 patients who underwent reintervention due to pulmonary stenosis, there was no mortality and nine patients $(56 \%)$ had the intervention within two years following the surgery

(Figure 5). 14 patients had one Intervention, one patient underwent two interventions, and one patient underwent three interventions.

Furthermore, 6 out of the 16 patients had simple TGA, and 10 had complex TGA.

The common type of pulmonary stenosis was supra-valvular and was observed in (14 out of 16), and only two patients had valvar and sub-valvar pulmonary stenosis. In the supra-valvular group, three patients had main pulmonary artery stenosis, three patients had right pulmonary artery stenosis, one patient had left Pulmonary artery stenosis and seven patients had bilateral pulmonary artery stenosis. The clinical decision to perform the reintervention was based on the multidisciplinary (heart-team) meeting.

Out of the 19 reinterventions performed (19 reinterventions for 16 patients), 12 were surgical, Figure 6.

Longitudinal Data Analysis

All serial transthoracic echocardiographic studies, excluding the intraoperative TEE for all

patients, were included $(\mathrm{N}=1844)$. Longitudinal graphical data display was used, respecting the

different timing of the echocardiographic measurements.

The averaged longitudinal data for the total gradient showed that most of the changes happen in the first two years following surgery (Figure 7).

Longitudinal Data Analysis with stratification:

Stratification by the sex, type of transposition, balloon atrial septostomy and the operating surgeon did not show significant differences. The most notable finding in the stratified longitudinal data display was the rate of drop of the pressure gradient between those who underwent reintervention and those who did not (Figure 8). Longitudinal data analysis with mixed effect modeling showed that the significant variables in relation to the peak pressure gradient following arterial switch operation included: younger age at surgery, complex transposition, and longer bypass time.

As the rate of change of pressure gradient immediately after surgery was the most notable difference between patients based on reintervention. Further testing with proportional hazard modeling for all variables with time-to-reintervention as an outcome with stepwise selection methodology showed that the only predictive preoperative variable for reintervention was the total peak gradient $(\mathrm{MPA}+\mathrm{RPA}+\mathrm{LPA})(\mathrm{p}<0.0001)$. Further analysis with the area under the receiver operating characteristics curve showed that a total peak gradient $55 \mathrm{mmHg}$ or more was a predictor for reintervention with a hazard ratio of 12 .

\section{Discussion:}

There has been attention to the neo-pulmonary artery stenosis after arterial switch operation as it is the commonest reason for reintervention ${ }^{3-5}$. Therefore, several techniques to reconstruct the pulmonary artery 
during arterial switch procedures have been developed, including pantaloon pericardial patch, limited buttons resection, and extended pericardial patch to pulmonary branches.

The use of pantaloon pericardial patch compared to synthetic patch was associated with lower neo-pulmonary artery stenosis ( $7 \%$ versus $40 \%$ ) and reintervention rate (4\% versus 14\%), respectively. However, complex anatomy increased risk for neo-pulmonary reintervention ${ }^{4}$.

Regarding Limited buttons resection technique, a data of 120 patients and follow up $66 \pm 46$

Months revealed that $(5 \%)$ of the patients required neo-pulmonary reintervention ${ }^{5}$.

A report by Wervovsky and colleagues was in favor of extended pericardial patch technique due to the result of gradual recognition of the necessity to avoid tension on the anastomosis of the neo-pulmonary artery. This led to the more extensive mobilization of the distal pulmonary arteries and the use of more generous pericardial patches (single or double), not only for filling in the defects created by coronary arteries dissection but also to add length to the proximal neo-pulmonary root ${ }^{7}$.

Pulmonary artery reconstruction with quadrangular fresh autologous pericardial patch represents a practical, simple and reproducible option during the arterial switch operation with all positions of the great vessels. The incidence of postoperative pulmonary stenosis is low, and there may be considerable potential for unlimited tissue growth. The peak pressure gradient across the neo-pulmonary valve and pulmonary artery branches as determined by transthoracic echocardiography is the most common clinical tool to assess the outcome of the surgical repair. Ideal results would show laminar flow across the proximal pulmonary tree with no significant pressure gradient. However, there is almost always an element of flow acceleration and measured pressure gradient that could be attributed to stretching of the pulmonary arteries secondary to Lecompte maneuver and the reconstruction technique of the neo-pulmonary artery. The value of that residual pressure gradient is ill-defined in the literature. This paper is focused on examining the value of the residual pressure gradient, its behavior over time, and its relationship with the need for reintervention.

In this report, 309 patients were included with all echocardiographic measurements that were performed in standardized fashion $(\mathrm{n}=1844)$. The rate of reintervention was $5 \%$, which is at the lowest part of the published ranges ${ }^{5,8-11}$. There was no difference in the pressure gradient in the presence or absence of an associated ventricular septal defect. It was not surprising to find the residual pressure gradient more in patients with complex anatomy and longer bypass time as compared to those without.

Our institutional repair strategy is based on using a fresh autologous redundant pericardial patch for the reconstruction of the neo-pulmonary artery. Furthermore, the impact of the surgeon was not significant in predicting the pressure gradient or reintervention in multivariable testing. The most notable difference in pressure gradient was seen in the first postoperative transthoracic echocardiogram (pre-discharge). Using the longitudinal data analysis with stratification based on intervention, it showed clear demarcation of the behavior of the pressure gradient. We propose if the pressure gradient as measured in the first postoperative transthoracic echocardiogram is $55 \mathrm{mmHg}$ or more will likely increase over time and subsequently require re-intervention. The threshold for reintervention was a clinical decision was based on the observed increase in the pressure gradient across the pulmonary arteries. The sustainability of laminar flow in the main pulmonary artery and its branches is the main component of maintaining low longitudinal averaged mean line of the total peak gradient following surgery for the entire follow up time (17-year), and we believe that enough dissection of right and left pulmonary arteries and tension-free anastomosis with redundant fresh pericardial patch for the reconstruction of the neo-pulmonary artery are crucial to achieving this result.

Additional statistical techniques were used to determine the best cut-off point of the "high gradient" that will persist, get higher and predict reintervention. The best cut-off point was a total peak gradient across the neopulmonary artery, right pulmonary artery and left pulmonary artery as measures in the first postoperative echocardiography of $55 \mathrm{mmHg}$ or higher.

The association between the transposition of the great arteries and pulmonary artery growth has been suggested in published reports relating it to accelerated pulmonary vasculopathy and asymmetric distribution 
of the pulmonary blood flow ${ }^{12,13}$.

\section{Conclusion:}

The total peak gradient (across pulmonary valve, right and left pulmonary artery branches) measured in the early postoperative echocardiogram is the most significant predictor for reintervention after arterial switch operation. Furthermore, we propose that the total peak gradient in the early postoperative echocardiogram of $55 \mathrm{mmHg}$ or more is a predictor for reintervention.

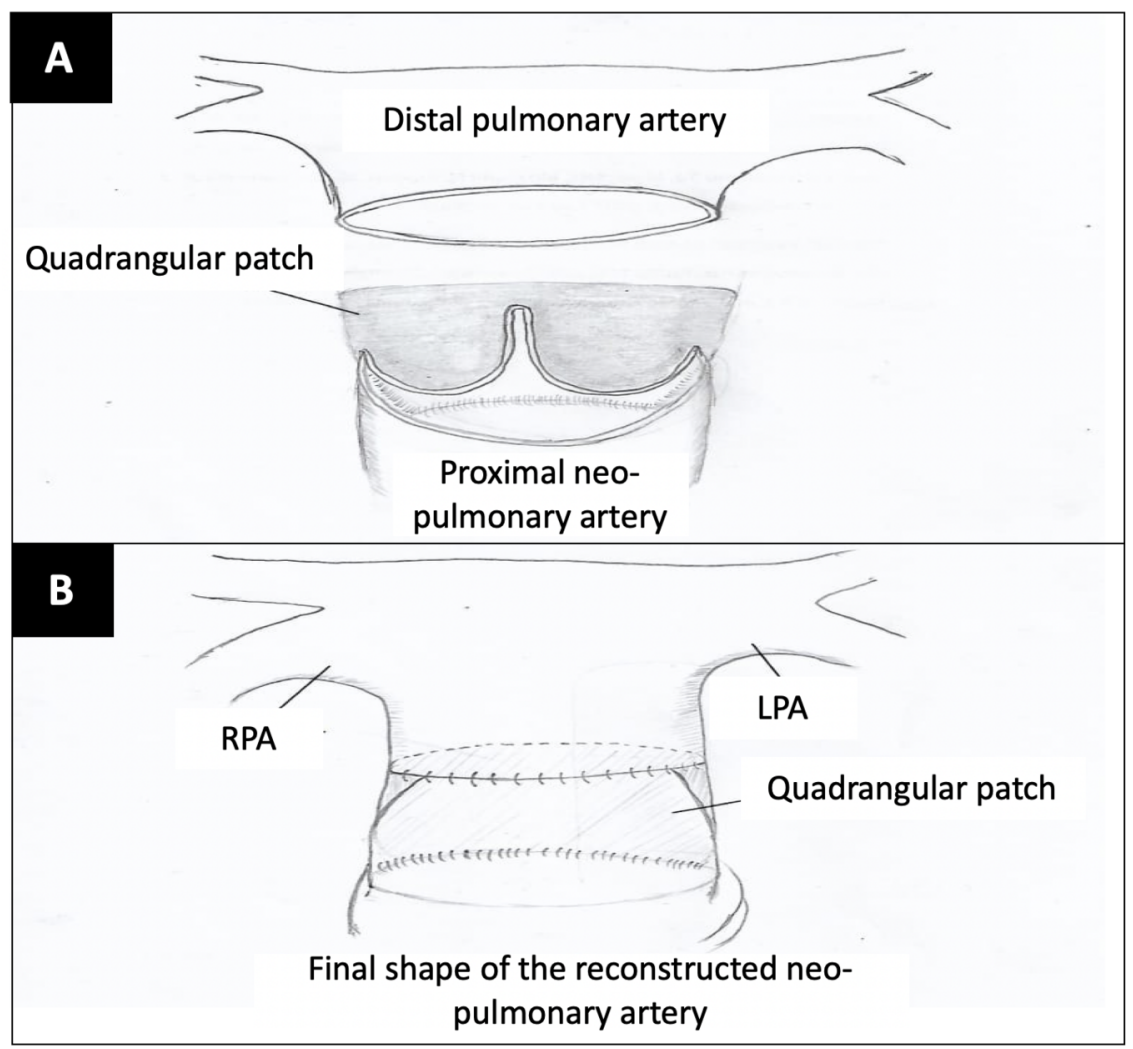

Figure 1: Illustration of the surgical technique 


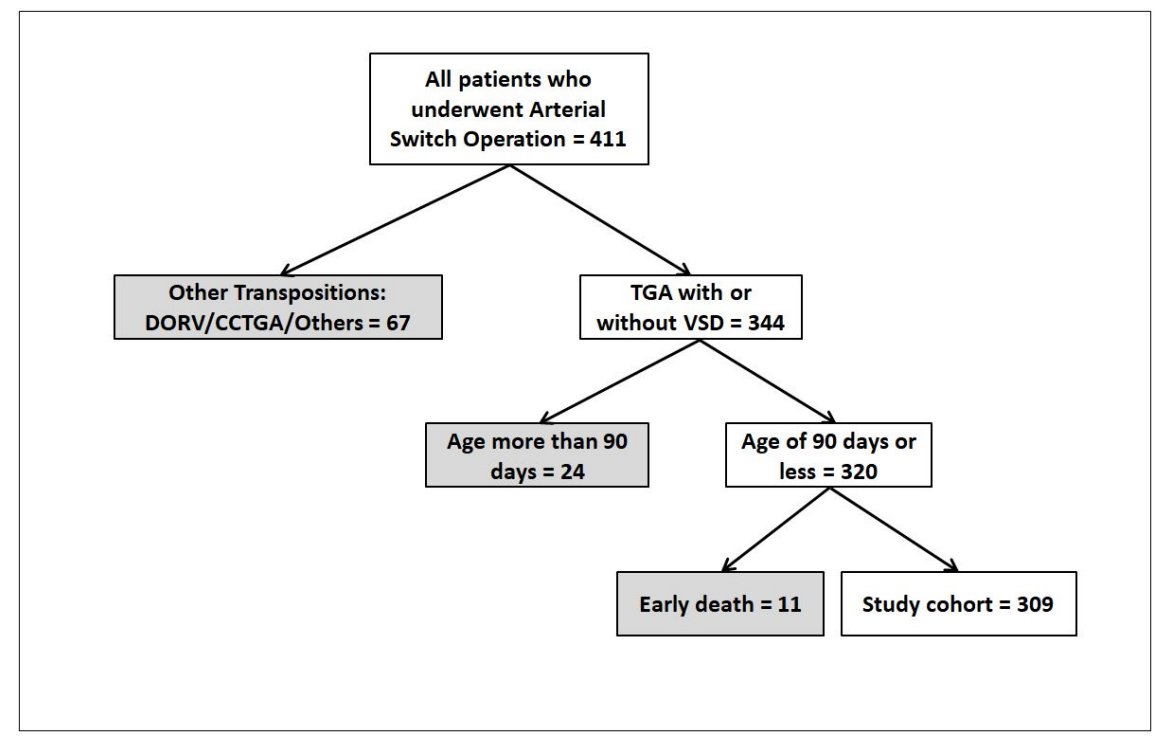

Figure 2: Patients' selection based on inclusion/exclusion criteria

Table 1: The general characteristics of the entire cohort of patients $N=309$

\begin{tabular}{ll}
\hline Variable & Number (Proportion) \\
Sex Male Female & $109(35 \%) 200(65 \%)$ \\
Type Simple transposition Complex transposition & $201(65 \%) 108(35 \%)$ \\
Balloon atrial septostomy & $171(55 \%)$ \\
Reintervention for residual pulmonary artery stenosis & $16(5 \%)$ \\
\hline
\end{tabular}

Table 2: The general characteristics of the entire cohort of patients $N=309$

\begin{tabular}{ll}
\hline Variable & Mean \\
Age (day) & $20 \pm 17$ \\
Weight $(\mathrm{kg})$ & $3.2 \pm 0.5$ \\
Height $(\mathrm{cm})$ & $51 \pm 5$ \\
Body surface area $\left(\mathrm{m}^{2}\right)$ & $0.2 \pm 0.02$ \\
Cardiopulmonary bypass time (min) & $160 \pm 39$ \\
Cross clamp time (min) & $95 \pm 27$ \\
Peak pressure gradient across the neo-pulmonary valve $(\mathrm{mmHg})$ & $9 \pm 7$ \\
Peak pressure gradient across the left pulmonary artery $(\mathrm{mmHg})$ & $14 \pm 11$ \\
Peak pressure gradient across the right pulmonary artery $(\mathrm{mmHg})$ & $17 \pm 13$ \\
Total Peak Gradient* & $40 \pm 24$ \\
\hline
\end{tabular}

* the total peak pressure gradient measured simultaneously across the main pulmonary artery + the right and left pulmonary artery

Table 3: Univariable comparison based on reintervention

Variable

Age (day)
No Intervention $(\mathrm{N}=293) \quad$ Intervention $(\mathrm{N}=16$ $20 \pm 17$ 
Weight $(\mathrm{Kg})$

$3.2 \pm 0.5$

$3.3 \pm 0.6$

Height $(\mathrm{cm})$

$51 \pm 5$

$51 \pm 4$

Body surface area $\left(m^{2}\right)$

$0.2 \pm 0.02$

$0.2 \pm 0.03$

Cardiopulmonary bypass time (min)

$160 \pm 39$

$182 \pm 48$

Cross clamp time (min)

$96 \pm 27$

$101 \pm 28$

Peak pressure gradient across the neo-pulmonary valve $(\mathrm{mmHg}) \quad 9 \pm 6$

$14 \pm 13$

Peak pressure gradient across the left pulmonary artery $(\mathrm{mmHg})$

$13 \pm 9$

$28 \pm 25$

Peak pressure gradient across the right pulmonary artery $(\mathrm{mmHg})$

$16 \pm 11$

$33 \pm 24$

Total Peak Gradient*

$38 \pm 20$

$76 \pm 46$

Table 4: Univariable comparison based on reintervention

\begin{tabular}{llll}
\hline Variable & No Intervention (N=293) & Intervention (N=16) & p-value \\
Male & $104(35 \%)$ & $5(31 \%)$ & 0.7 \\
Complex transposition & $100(93 \%)$ & $8(7 \%)$ & 0.2 \\
Balloon atrial septostomy & $160(94 \%)$ & $11(6 \%)$ & 0.3 \\
Surgeon A & $97(96 \%)$ & $4(4 \%)$ & 0.8 \\
Surgeon B & $158(94 \%)$ & $10(6 \%)$ & 0.8 \\
Surgeon C & $38(95 \%)$ & $2(5 \%)$ & 0.8 \\
\hline
\end{tabular}

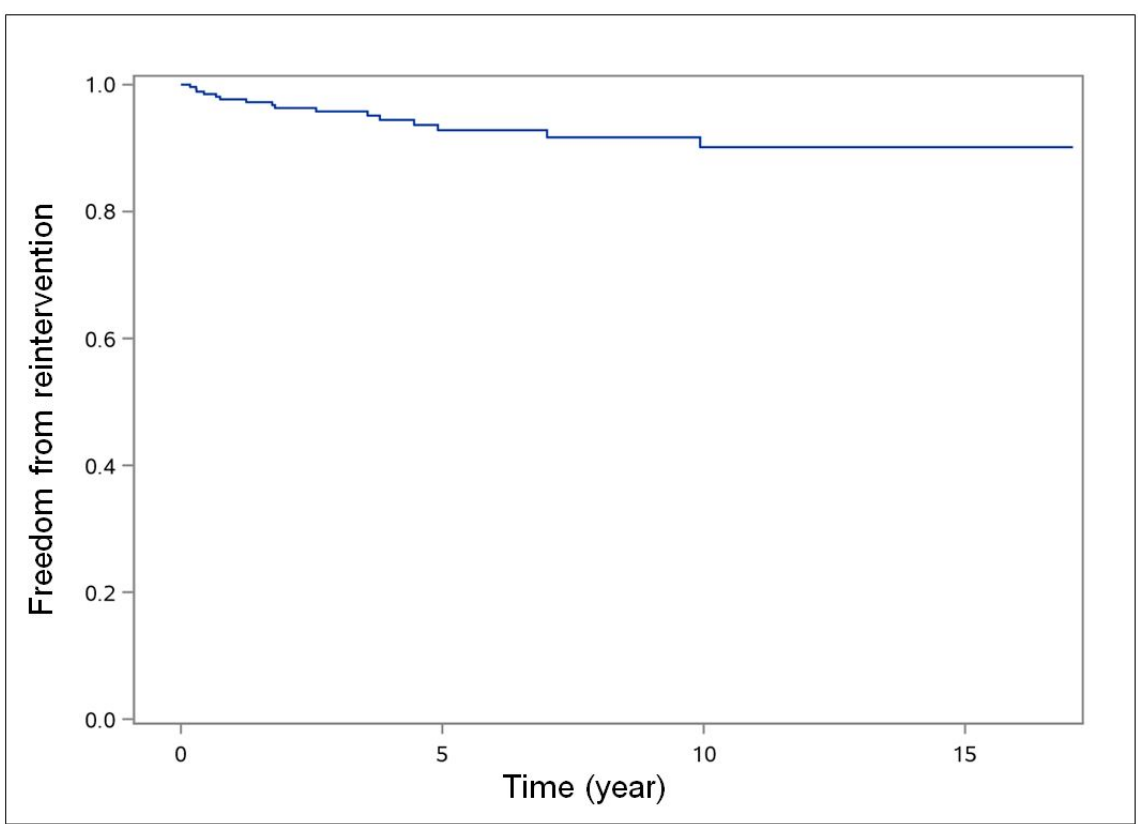

Figure 3: Kaplan-Meier Curves for the entire cohort (time-to-reintervention) 


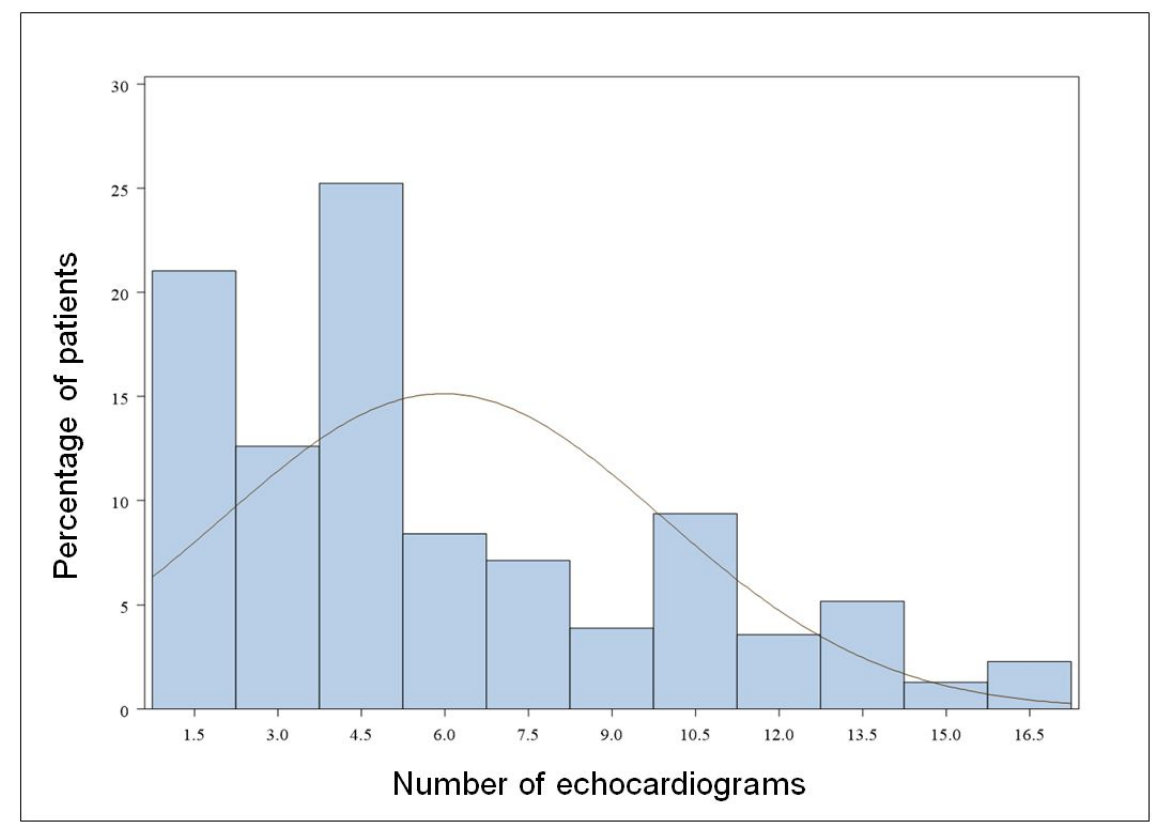

Figure 4: Histogram displaying the number of transthoracic echocardiograms in relation to patients

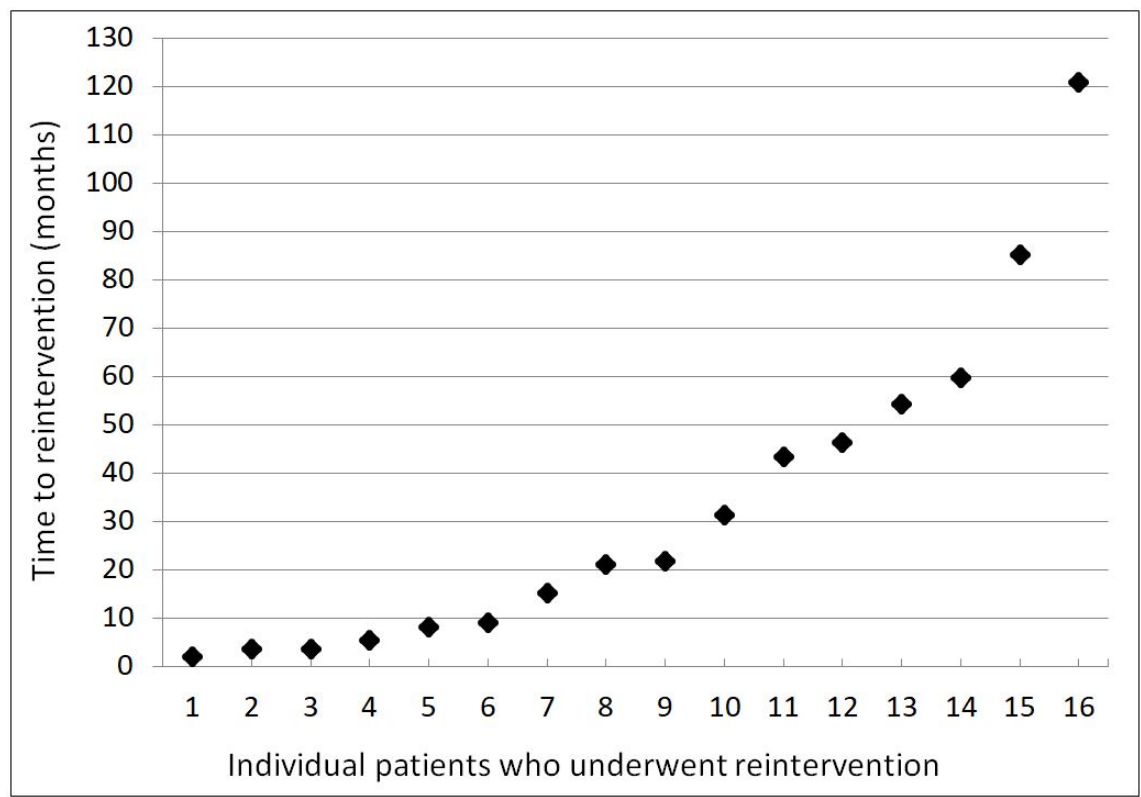

Figure 5: Time to reintervention $(\mathrm{N}=16)$ 


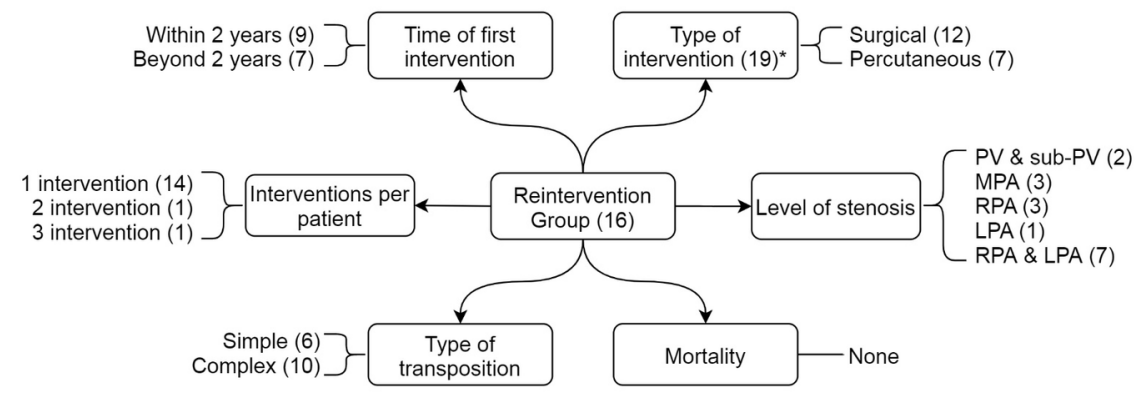

* Total number of interventions performed for the 16 patients

Figure (6): Re-intervention group of patients

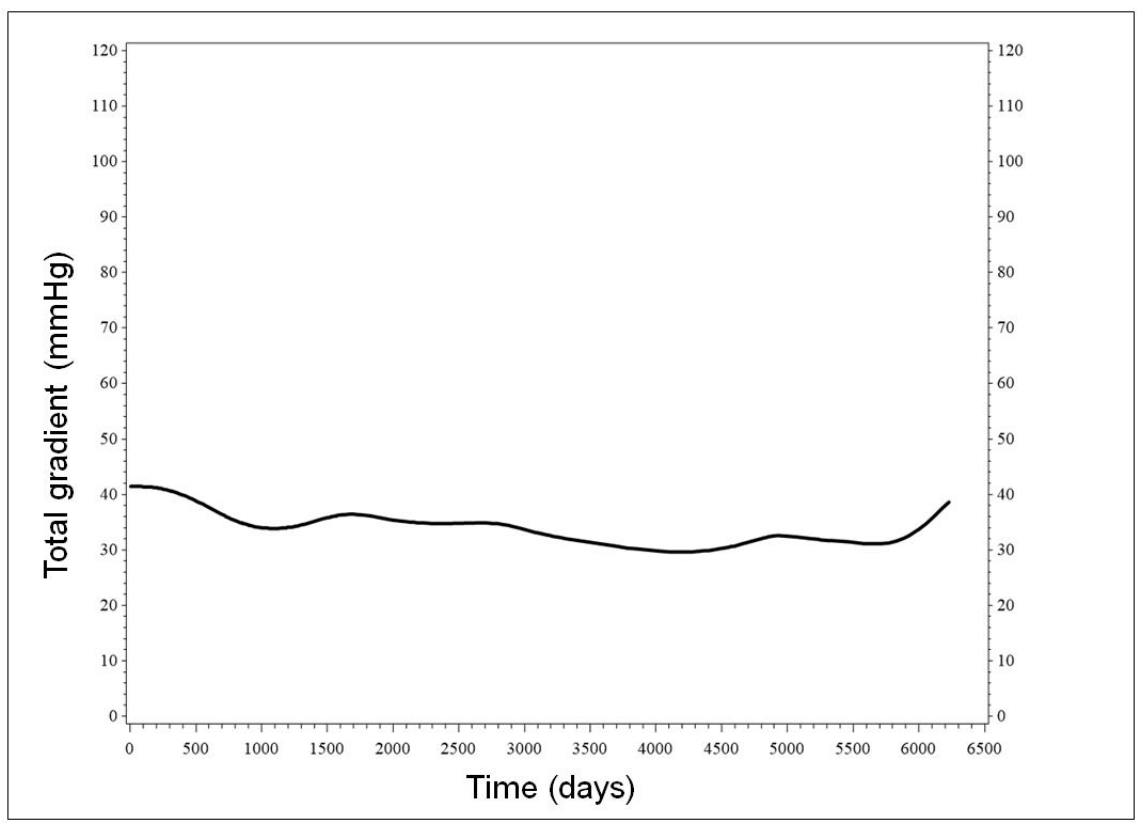

Figure 7: Longitudinal averaged a mean line of the total gradient following surgery for the entire follow up time (17-year) 


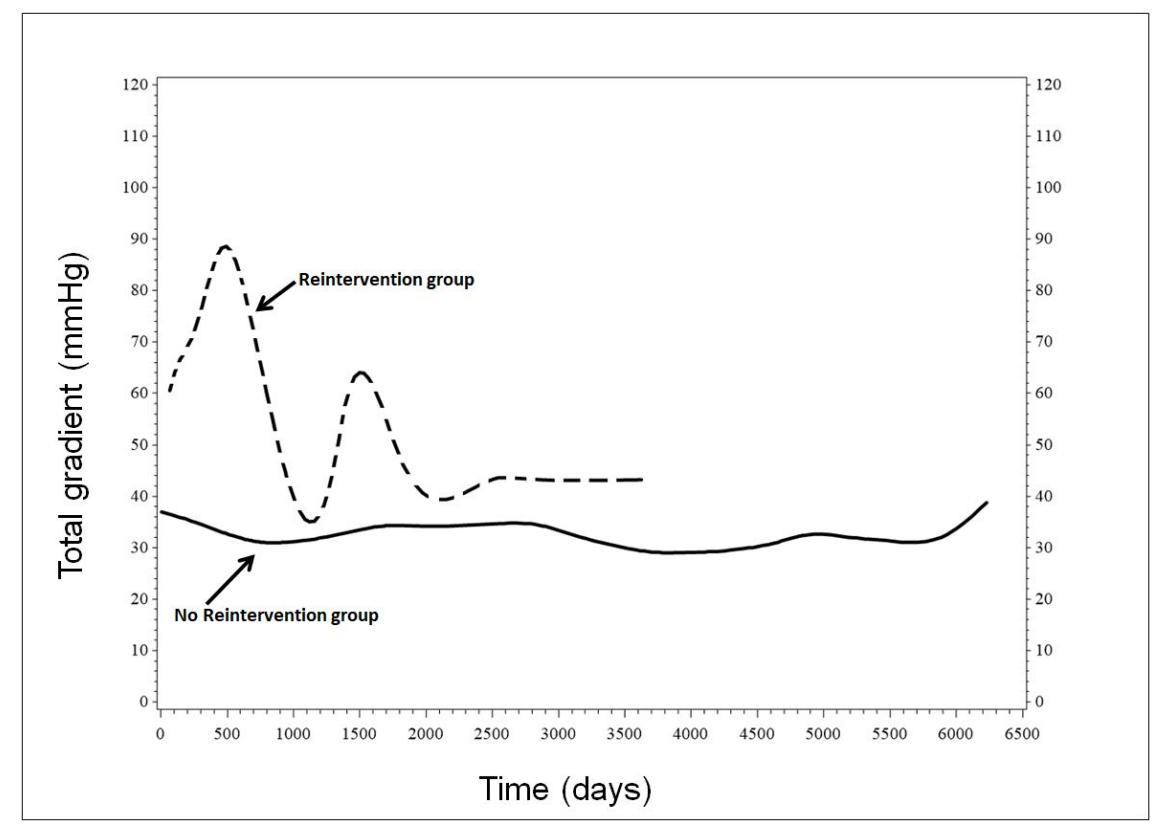

Figure 8: Longitudinal averaged the mean line of the peak pressure gradient following surgery for the re-intervention group.

References

1. Castaneda AR, Trusler GA, Paul MH, Blackstone EH, Kirklin JW. The early results of treatment of simple transposition in the current era. J Thorac Cardiovasc Surg 1988;95:14-28.

2. Jatene AD, Fontes VF, Paulista PP, et al. Anatomic correction of transposition of the great vessels. J Thorac Cardiovasc Surg 1976;72:364-70.

3. Losay J, Touchot A, Serraf A, et al. Late outcome after arterial switch operation for transposition of the great arteries. Circulation 2001;104:I121-6.

4. Rudra HS, Mavroudis C, Backer CL, et al. The arterial switch operation: 25-year experience with 258 patients. Ann Thorac Surg 2011;92:1742-6.

5. Swartz MF, Sena A, Atallah-Yunes N, et al. Decreased incidence of supravalvar pulmonary stenosis after arterial switch operation. Circulation 2012;126:S118-22.

6. Lecompte Y, Zannini L, Hazan E, et al. Anatomic correction of transposition of the great arteries. J Thorac Cardiovasc Surg 1981;82:629-31.

7. Wernovsky G, Mayer JE, Jr., Jonas RA, et al. Factors influencing early and late outcome of the arterial switch operation for transposition of the great arteries. J Thorac Cardiovasc Surg 1995;109:289301 ; discussion -2 .

8. Michalak KW, Moll JA, Sobczak-Budlewska K, et al. Reoperations and catheter interventions in patients with transposition of the great arteries after the arterial switch operation. Eur J Cardiothorac Surg 2017;51:34-42.

9. Nakanishi T, Matsumoto Y, Seguchi M, Nakazawa M, Imai Y, Momma K. Balloon angioplasty for postoperative pulmonary artery stenosis in transposition of the great arteries. J Am Coll Cardiol 1993;22:85966. 
10. Nellis JR, Turek JW, Aldoss OT, Atkins DL, Ng BY. Intervention for Supravalvar Pulmonary Stenosis After the Arterial Switch Operation. Ann Thorac Surg 2016;102:154-62.

11. Hutter PA, Kreb DL, Mantel SF, Hitchcock JF, Meijboom EJ, Bennink GB. Twenty-five years' experience with the arterial switch operation. J Thorac Cardiovasc Surg 2002;124:790-7.

12. Aziz KU, Paul MH, Rowe RD. Bronchopulmonary circulation in d-transposition of the great arteries: possible role in genesis of accelerated pulmonary vascular disease. Am J Cardiol 1977;39:432-8.

13. Muster AJ, Paul MH, Van Grondelle A, Conway JJ. Asymmetric distribution of the pulmonary blood flow between the right and left lungs in d-transposition of the great arteries. Am J Cardiol 1976;38:352-61. 